\title{
Repair of abdominal fascial defects caused by trauma-related infection
}

\author{
Seung Je Go, Yun Su Mun, Seung Ho Bang \\ Department of Trauma Surgery, Trauma Center of Eulji University Hospital, Daejeon, Korea
}

Received May 13, 2020

Revised June 9, 2020

Accepted June 9, 2020

Correspondence to

Seung Je Go

Department of Trauma Surgery,

Trauma Center of Eulji University

Hospital, 95 Dunsanseo-ro, Seo-gu,

Daejeon 35233, Korea

Tel: +82-42-611-3429

Fax: +82-42-611-3858

E-mail:rhtmdwp@gmail.com
Abdominal fascial defects can be caused by a congenital disorder, being older, or complications of previous abdominal surgery. The timing and procedure of abdominal fascial defect repair depend on the time of its diagnosis, its size and symptoms, and the presence of associated intra- and extra-abdominal lesions. However, in case of an abdominal fascial defect caused by a trauma-related infection, it may be more difficult to determine when and how to repair the defect because of additional problems related to the trauma. We reported the cases of two patients who underwent such repair using a porcine-derived acellular dermal sheet for planned ventral hernia repair in abdominal fascial defects caused by a trauma-related infection.

Key Words: Trauma, Infections, Ventral hernia

\section{Introduction}

Abdominal fascial defect repair is a common surgical procedure, but challenging complications may occur sometimes. Abdominal fascial defect repair's timing and procedure depend on its diagnosis time, size and symptoms, and presence of associated intra- and extra-abdominal lesions. For large abdominal fascial defects due to trauma-related infections, determining the timing and procedure of the repair can be more difficult because of additional problems, including contamination, ongoing bleeding, and other trauma-related injuries. We reported the cases of two patients who underwent such repair using a porcine-derived acellular dermal sheet $\left(\right.$ Permacol $\left.^{\mathrm{TM}}\right)$ for planned ventral hernia in a large abdominal fascial defect following repeated intra-abdominal and abdominal wall infection after laparotomy, which led to severe abdominal contamination.

\section{Case presentation}

\section{Case 1}

A 43-year-old male was referred to our emergency department from a local hospital because of a traffic accident that led to his abdomen being stuck in the steering wheel 2 days ago. Sigmoid colon perforation with an anterior abdominal wall injury and fecal contamination with a small fascial defect was diagnosed. Emergency Hartmann's procedure was immediately performed with abdominal wall wound drainage. His general condition was tolerable, but abdominal surgical wound worsened with widening necrosis of the lower abdominal fascia. We managed to repeat abdominal wound opening and closure to control infection and performed delayed repair of planned ventral

(C)This is an Open Access article distributed under the terms of the Creative Commons Attribution Non-Commercial License (http://creativecommons.org/licenses/by-nc/4.0) which permits unrestricted noncommercial use, distribution, and reproduction in any medium, provided the original work is properly cited.

Copyright (c) 2020 Korean Association for Research, Procedures and Education on Trauma. All rights reserved. 
hernia.

After 3 months, we repaired the abdominal wall hernia by concurrent colostomy reversal. Preoperative abdominal computed tomography (CT) (Fig. 1) revealed a large fascial defect in the anterior abdominal wall (approximately $14 \times 11 \mathrm{~cm}$ ) (Fig. 2). We performed abdominal fascial defect repair using a porcine-derived acellular dermal sheet $\left(\right.$ Permacol $^{\mathrm{TM}}$, Covidien, Mansfield MA, USA, $15 \times 20 \mathrm{~cm}$ ) (Fig. 3) owing to the large-sized defect and possibility of inadvertent surgical site infection.

\section{Case 2}

A 15-year-old male presented to the emergency department with severe abdominal injury after falling over wires for defense hanging over a truck while riding a bicycle. $\mathrm{He}$ suffered eviscerated small bowel and colon through a large open abdominal wound (approximately 25 -cm length). Following CT, we performed immediate emergency oper-
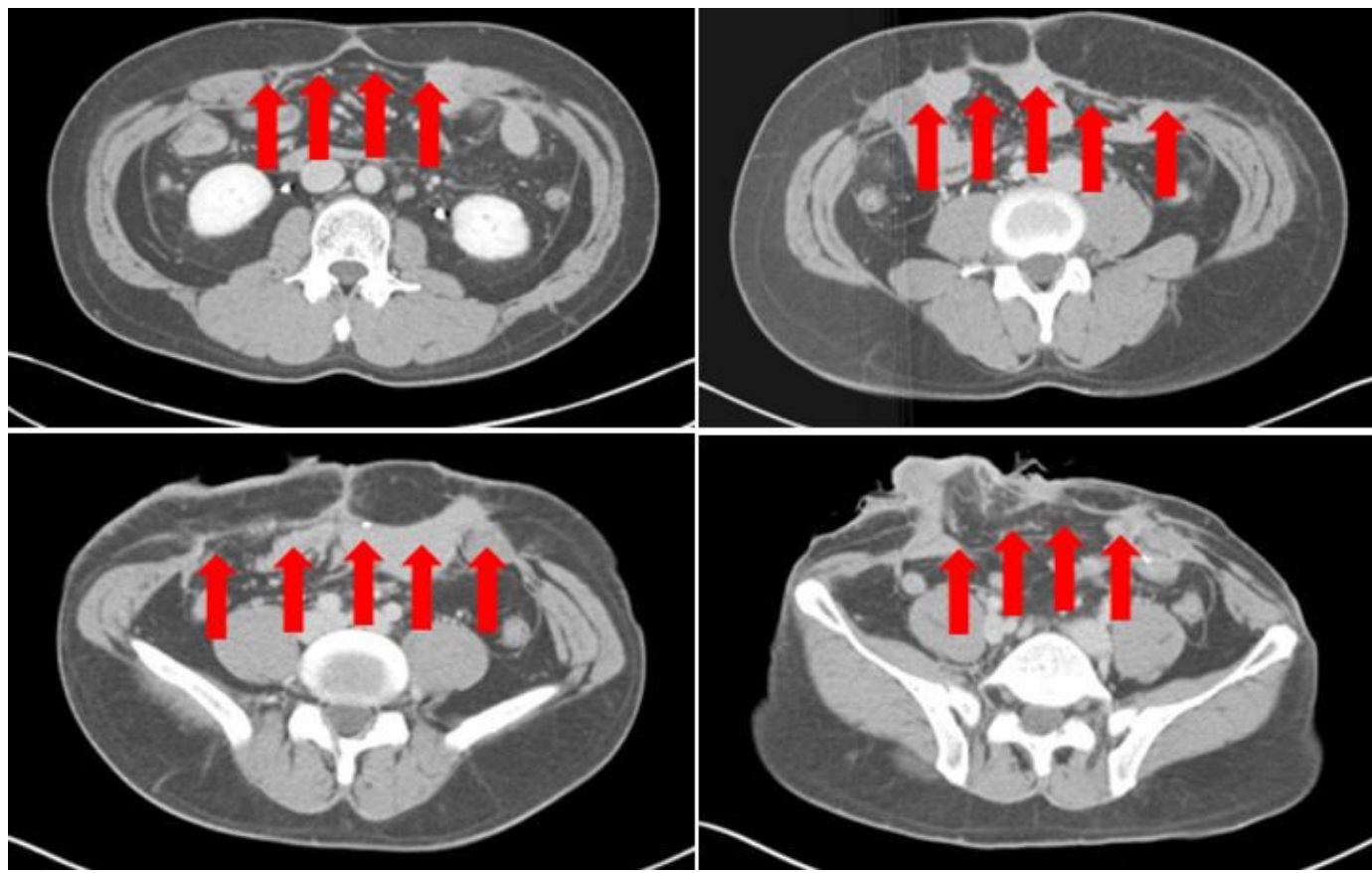

Fig. 1. Abdominal computed tomography shows an approximately $14 \times 11-\mathrm{cm}$ fascial defect in the anterior abdominal wall (red arrows).
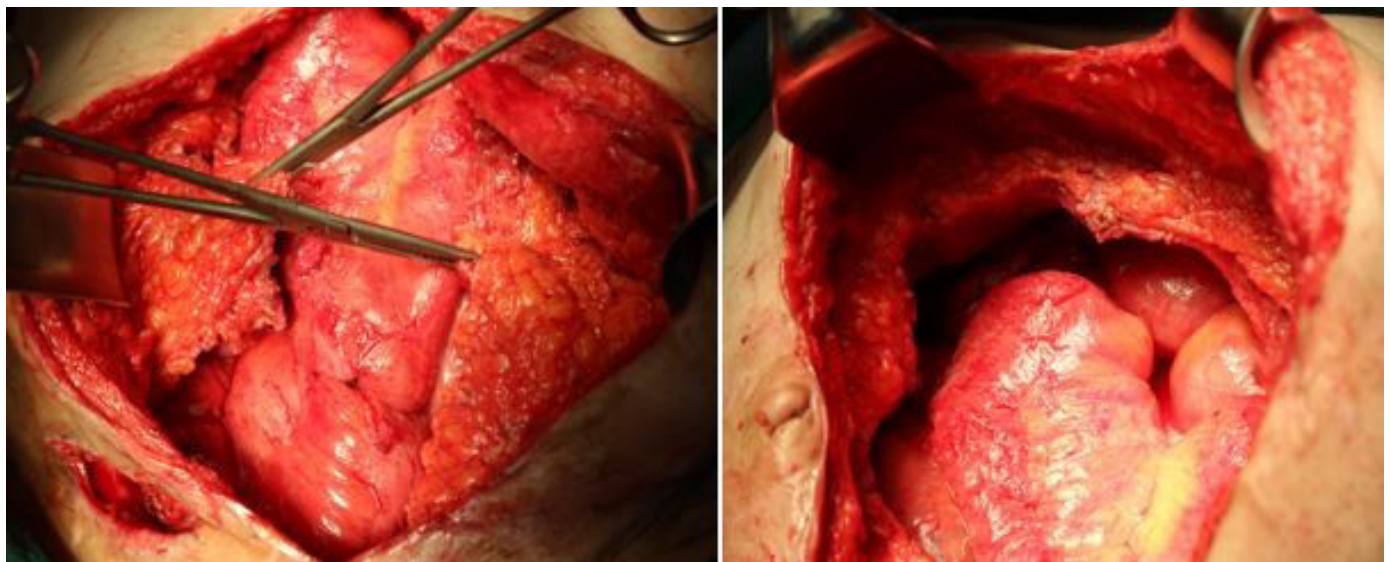

Fig. 2. A $14 \times 11-\mathrm{cm}$ fascial defect in the anterior abdominal wall. 

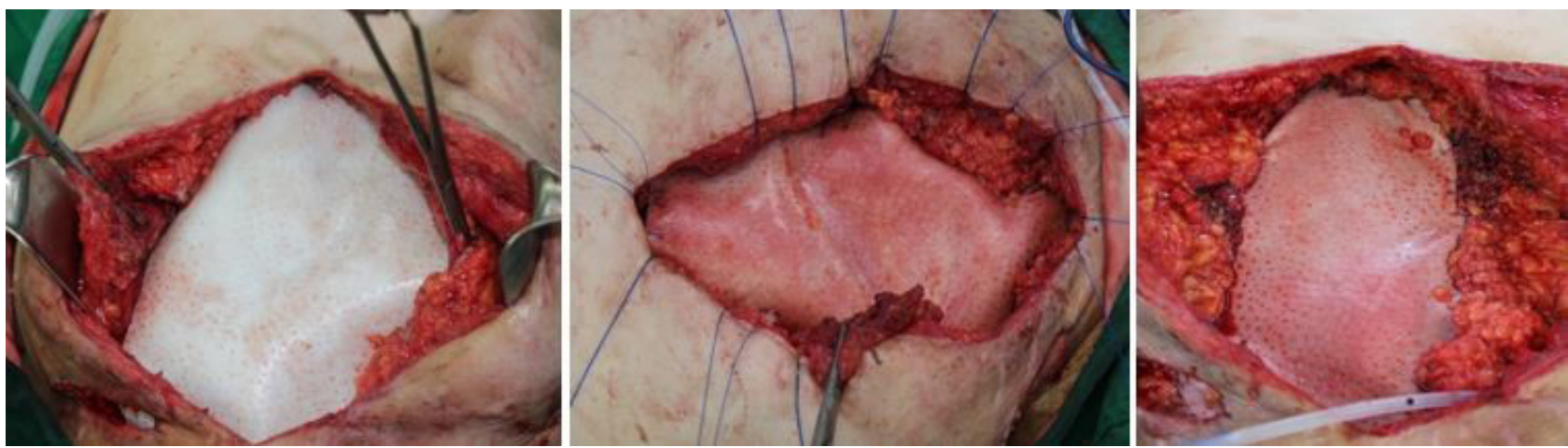

Fig. 3. Repair of the large abdominal fascial defect using a porcine-derived acellular dermal sheet $\left(\right.$ Permacol $\left.^{\mathrm{TM}}\right)$ with nonabsorbable suture.
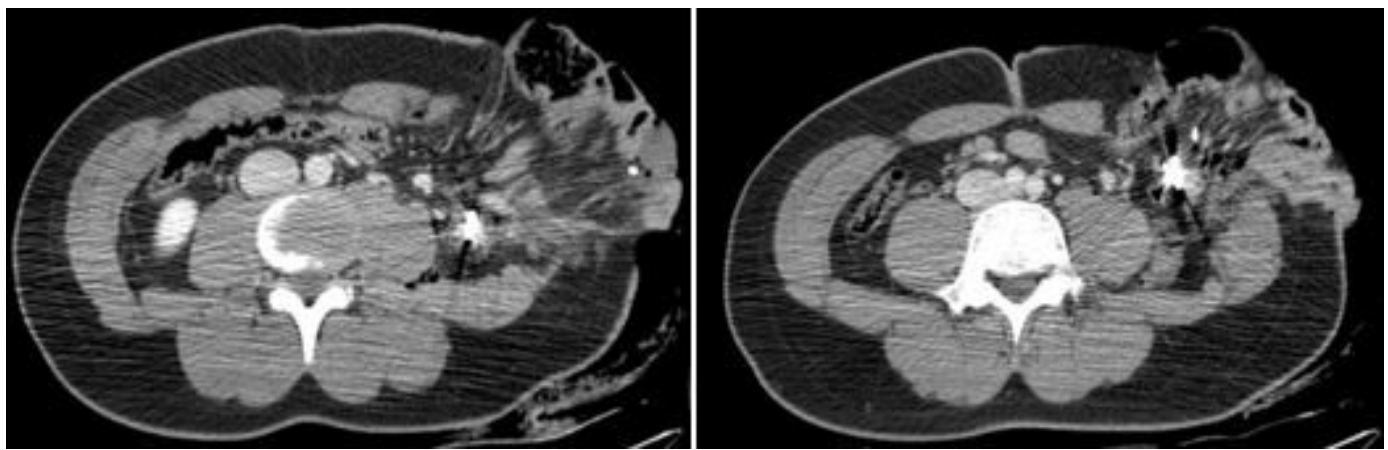

Fig. 4. Abdominal computed tomography shows small bowel and left large bowel evisceration with a wide abdominal fascia defect.

ation (Fig. 4).

Emergency laparotomy revealed small bowel and left large bowel injuries with a wide abdominal fascial defect caused by the wires. Approximately $150-\mathrm{cm}$ of the injured jejunum with severe mesenteric laceration and multiple perforations was resected. Left hemicolectomy was performed due to a descending colon injury involving full thickness transection and devascularization. Temporary abdominal closure was performed for planned delayed closure due to severe intra- and extra-abdominal contamination. After 2 days, anastomosis of the resected jejunum and end-colostomy were performed with primary closure of the approximately $9 \times 8-\mathrm{cm}$ abdominal wall fascial defect. Thereafter, several episodes of wound infection with fascitis around the abdominal wound occurred; thus, we performed planned ventral hernia repair.

After 3 months, we performed colostomy reversal and definitive fascial defect closure using a porcine-derived acellular dermal sheet $\left(\right.$ Permacol $\left.{ }^{\mathrm{TM}} 10 \times 10 \mathrm{~cm}\right)($ Fig. 5).

\section{Discussion}

Abdominal fascial defects can be caused by various factors such as trauma. Abdominal wall musculature and fascial injuries may be caused by physical trauma to the abdomen or abdominal surgical complications after trauma. Without rapid correction, prolonged abdominal wall herniation may occur, progressing to an abdominal wall defect that becomes difficult to repair. Therefore, primary fascial closure should be achieved as soon as possible, but in some cases, it cannot or should not be attempted (1). In such cases, planned ventral hernia repair is a management strategy aimed at hernia repair at a later stage when it is safe, feasible, and tolerated by the patient (2).

Various methods of abdominal fascial defect repair have been attempted. Depending on the abdominal fascial defect's cause and size and various patient conditions, primary suture with local fascia or repair using a mesh made of various materials can be performed. 


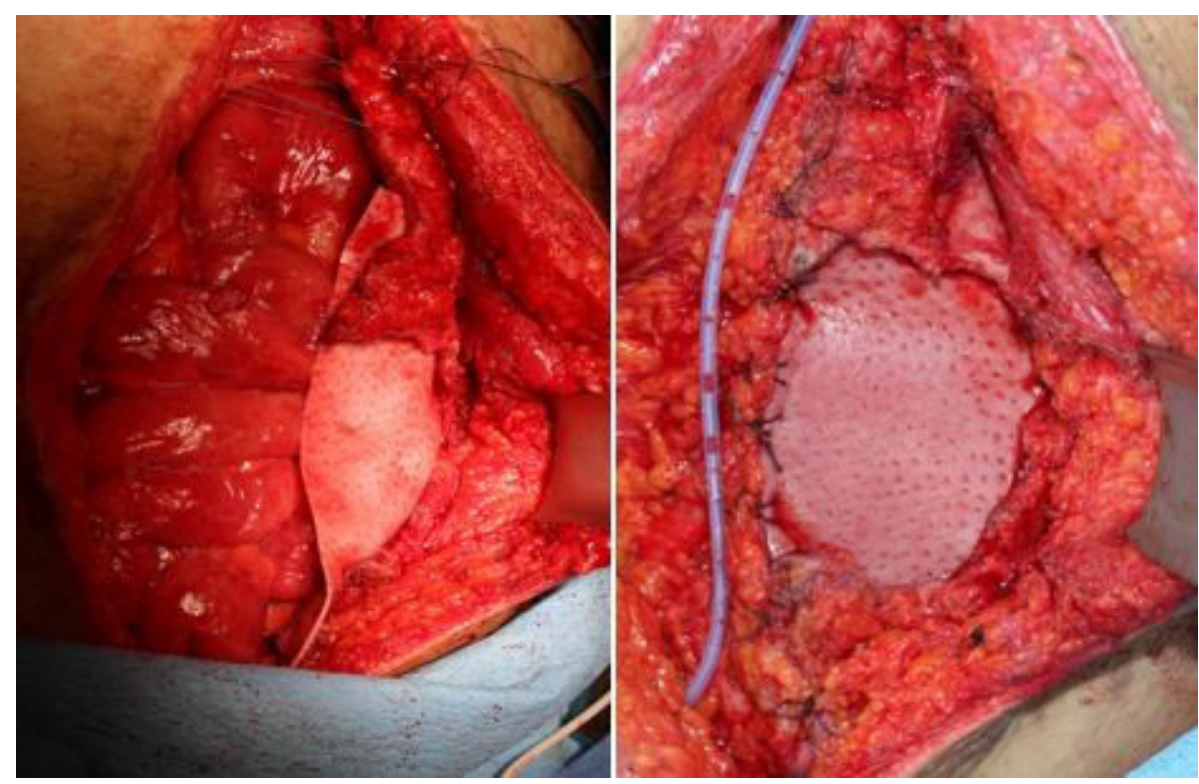

Fig. 5. Definitive closure of the fascial defect using a porcine-derived acellular dermal sheet (Permacol $\left.{ }^{\mathrm{TM}}\right)$.

In our cases, owing to large abdominal fascial defects due to trauma-related infections, primary repair using surrounding fascial tissue or an artificial mesh was difficult to perform. Thus, infection control was our first priority. We then planned ventral hernia repair for the abdominal fascial defect. Moreover, because abdominal fascial defect size and fascial injury around the defect were large, repair was performed using a biological mesh, i.e., a porcine-derived acellular dermal sheet $\left(\right.$ Permacol $\left.^{\mathrm{TM}}\right)$, with fewer complications than an artificial mesh.

Permacol $^{\mathrm{TM}}$ is an acellular porcine dermal collagen matrix with a cross-linked pattern. It can be used on a contaminated surgical site and for intraperitoneal placement with fewer complications, such as fistula, even if a synthetic mesh cannot be applied due to a severe abdominal fascial defect (3). Furthermore, the recurrence rate is similar to that of other surgical methods (4).

For abdominal fascial defects caused by trauma-related infections, especially large defects, repair using Perma$\mathrm{col}^{\mathrm{TM}}$ is a good alternative. However, the cost remains burdensome and further studies are warranted as no long- term follow-up data are available.

\section{Conflict of interest}

No potential conflict of interest relevant to this article was reported.

\section{References}

1. Schein M. Surgical management of intra-abdominal infection: is there any evidence? Langenbecks Arch Surg. 2002;387:1-7.

2. Ari L, Erkki T. Planned hernia repair and late abdominal wall reconstruction. World J Surg. 2012;36:511-5.

3. Parker DM, Armstrong PJ, Frizzi JD, North JH Jr. Porcine dermal collagen (Permacol) for abdominal wall reconstruction. Curr Surg. 2006;63:255-8.

4. Janis JE, O’Neill AC, Ahmad J, Zhong T, Hofer SO. Acellular dermal matrices in abdominal wall reconstruction: a systematic review of the current evidence. Plast Reconstr Surg. 2012;130:183-93. 